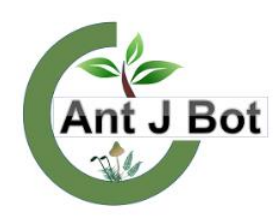

Received : 03.03.2018 Accepted : 30.08 .2018

\title{
Endemic plants of Lake Van Basin
}

\author{
Nasip DEMIRKUŞ ${ }^{1 *}$, Mehmet KOYUNCU ${ }^{2}$, Mesut GÜL ${ }^{3}$ \\ ${ }^{1}$ Yüzüncü Yll University, Education Faculty, Department of Mathematics and Science Education, Van, Turkey \\ ${ }^{2}$ Cyprus International University, Pharmacy Faculty, Lefkoşa, Turkish Republic of North Cyprus \\ ${ }^{3}$ Karabük University, Safranbolu Vocational High School, Karabük, Turkey \\ *nasip@hotmail.com
}

\section{Van Gölü Havzası endemik bitkileri}

\begin{abstract}
This study was carried out on endemic plants collected from the Lake Van basin between 1994 and 2002. As a result, 98 endemic taxa, 23 of which grow only in the Lake Van basin, were determined. Including the endemic taxa reported before from the region, a list of 259 endemic taxa belonging to 34 families and 117 genera were compiled. Twenty-three of 259 taxa listed, are unique to the Lake Van Basin. There are 249 endemic taxa in the VANF Herbarium. The authors collected 98 of them. The other 151 endemic taxa were determined by other researchers. Ten endemic taxa were recorded from the literature. In addition to the features in the Flora of Turkey, new features and variations of nine endemic taxa identified. Total 612 images and 200 pictures belonging to 98 endemic taxa scanned and transferred to computer. All images were uploaded to the web page, Virtual Herbarium of the Lake Van Basin, which is also a part of VANF Herbarium.

Key words: Endemic plants, taxonomy, IUCN, Turkey

Özet: Bu çalışma 1994-2002 yılları arasında Van Gölü havzasından toplanan endemik bitkiler üzerinde gerçekleştirilmiştir. Sonuçta, yirmi üç tanesi sadece Van Gölü havzasında yetişen, 98 endemik takson belirlenmiştir. Bölgeden daha önceden rapor edilen taksonların da dahil edilmesiyle, 34 familya ve 117 cins içinde yer alan 259 endemik taksondan oluşan bir liste oluşturulmuştur. Listelenen 259 taksondan 23 tanesi sadece Van Gölü Havzasına özgüdür. VANF Herbaryumu'nda 249 endemik takson vardır. Bunlardan 98 tanesi yazarlar tarafından toplanmıtır. Kalan 151 endemik takson ise diğer araştırmacılar tarafından belirlenmiştir. On endemik takson da literatürden kaydedilmiştir. Türkiye Florasındaki özelliklere ek olarak, dokuz endemik taksonun yeni özellikleri ve varyasyonları belirlenmiştir. Doksan sekiz endemik taksona ait 612 görüntü ve 200 resim camlı tarayıcıdan taranarak bilgisayar ortamına aktarılmışıtır. Bütün görüntüler VANF Herbaryum'unun bir parçası da olan web sayfasına, Van Gölü Havzası'nın Sanal Herbaryumu'na, yüklenmiştir.
\end{abstract}

Anahtar kelimeler: Endemik bitkiler, taksonomi, IUCN, Türkiye

\section{Introduction}

Floristic studies indicate that Turkey has a very rich species diversity. This is because the country is located in the transition area of three, Irano-Turanien, Mediterranean and Euro-Siberian, phytogeographical regions (Bulut and Y1lmaz, 2010). About 12.000 seed plant taxa have been identified in Turkey while this number is about 13.000 in whole of Europe (Erik ve Tarıkahya, 2004; Ekim, 2005). Turkey is also among the most important countries in terms of endemic plant species (Davis et al., 1988; Ekim, 2005; Özhatay et al., 2009). More than 3000 endemic plant taxa, most of which are threatened, have so far been determined within the boundaries of Turkey (Atalay, 1997; Ekim et al., 2000) and the localities of almost 2900 endemic taxa have been mapped (Şenkul and Kaya, 2017).

The Lake Van is situated on the high plateaus of Eastern Anatolia, between $38^{\circ} 18^{\prime}-39^{\circ} 00^{\prime} \mathrm{N}$ latitudes and $42^{\circ} 17^{\prime}$ $43^{\circ} 39^{\prime} \mathrm{E}$ longitudes. The lake surface stands at $1648 \mathrm{~m}$ above sea level, with a surface area of $3574 \mathrm{~km}^{2}$ (Degens et al., 1984). The drainage basin of Lake Van covers an area of about $20.000 \mathrm{~km}^{2}$ (Çiftçi et al., 2008) within the boundaries of Bitlis and Van provinces and falls in B9 according to P.H. Davis' grid square system. The basin is surrounded by high volcanic mountains. The long term annual precipitation is $387.2 \mathrm{~mm}$ and annual average temperature is $9.4^{\circ} \mathrm{C}(\mathrm{mgm}, 2018)$. In general the basin is characterized by steppe vegetation, and forest and shrubby areas are generally dominated by Quercus sp.

The study aims to present a complete list of endemic plant taxa growing in Van Lake basin.

\section{Materials and Method}

Endemic plant samples, which were collected by the authors from the field, existing in Yüzüncü Y1l University Herbarium (VANF) herbarium or those, obtained from the literature make the material of this study.

Endemic plant samples were collected from Lake Van basin (Fig 1) between the years 1994 and 2002. During field studies, colour slides were taken and required ecological data were recorded. Then the plant samples were transferred to the herbarium and prepared as herbarium materials. Identification was performed with the help of Flora of Turkey and the East Agean Islands (Davis, 1965-1982). Later on, the existing plant samples in VANF herbarium were traced and endemic taxa a belonging to Van Lake basin were listed together with the necessary information about the taxa. Meanwhile the related literature (Behçet, 2001; Doğan and Akaydın, 2004; Özgökçe et al., 2005; Sutorý, 2005; Akan et al., 2008; Karabacak and Behçet, 2009; Dirmenci et al., 2010; Eker and Babaç, 2010; Yıldırım et al., 2011; Yıldız et al., 2011; İlçim et al., 2013; Vitek et al., 2014; Yıldırırm, 2014; Doğan et. al., 2015; Tekşen and Karaman, 2015; Firat and Y1ldı, 2016; Gültepe et al., 2016; Firat, 2017a,b; P1nar, 2017) concerning the endemic plant taxa of Van Lake basin were also traced, and an endemic plant list with 259 taxa was created. Collection localities are given in Table 1. The collected samples are kept in VANF.

The validity of Endemic plants names was checked mainly from The Plant List Version 1.1 (2018). If required The 
Euro + Med Plant Base (2018) and The International Plant Names Index (IPNI) were used as secondary or tertiary sources.

\section{Results}

The compiled list of endemic plant taxa determined in the Lake Van basin is listed as cited in Flora of Turkey, together with their habitats, locality numbers, collection dates, voucher number(s), IUCN Red Data categories (if known) and the variations from the description given in Flora of Turkey (if determined). IUCN Red Data Categories and the abbreviations are in accordance with Ekim et al. (2000).

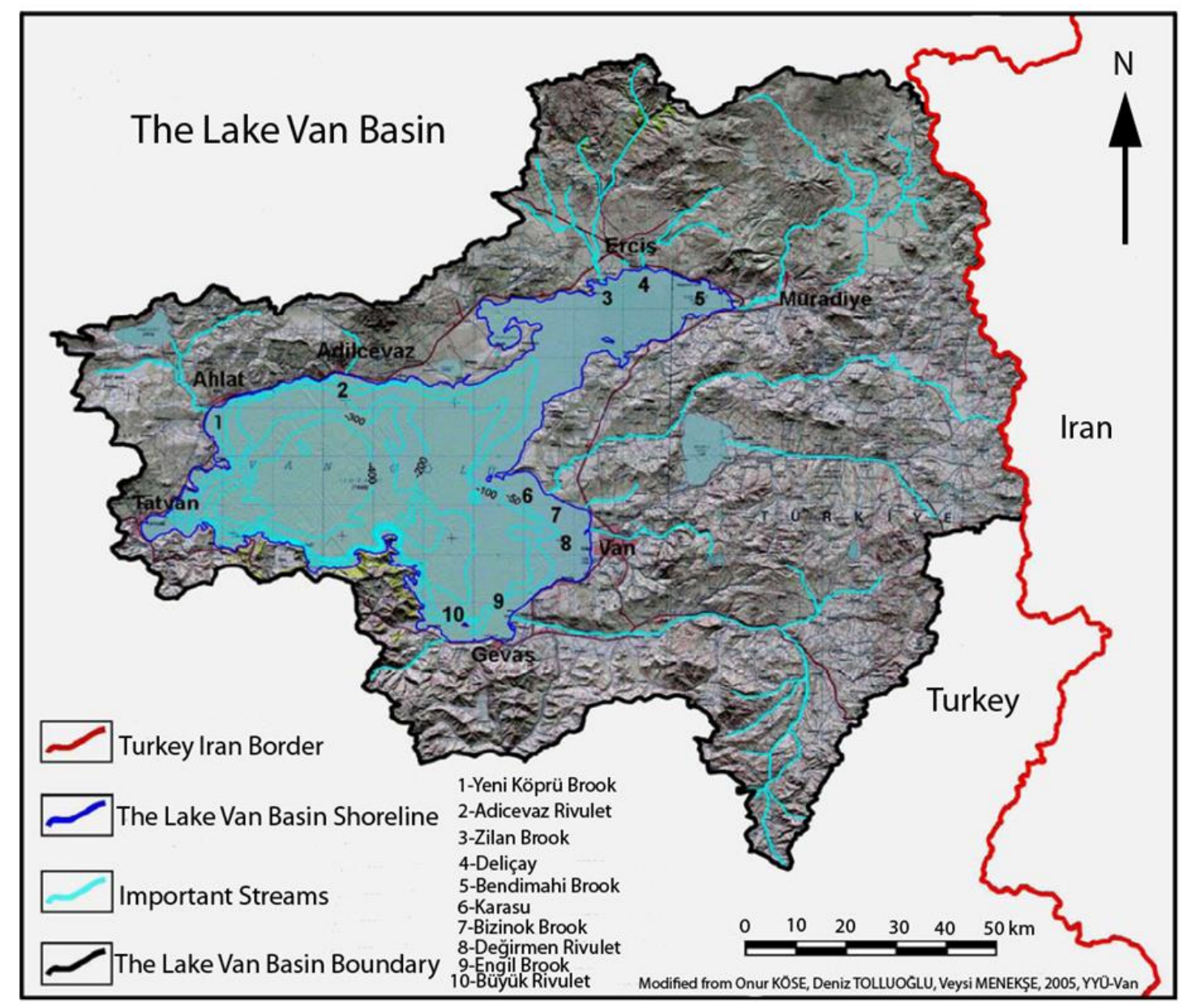

Figure 1. The hydrological border and the main drainage system of the Lake Van basin. (Modified from Çiftçi et al., 2008)

For The Citation Of The Voucher Numbers, Names Of The Collectors Are Abbreviated As Fallows: A.Altiok (Aa); Bayram Yildiz (Y); Davut Avlamaz (Da); Doğan \& Akaydin (D\&A); Ismail Eker (E); Fevzi Özgökçe (Fö); Hasan Özçelik (Hö); Hüseyin Eroğlu (He); H.Yildirim (Hy); Ibrahim Demir (Id); L.Behçet \& D. Avlamaz (B\&A); Lütfü Behçet (B); M. Gültep (Mg); Metin Armağan (Ma); Mehmet Firat (Mf); Mehmet Koyuncu (Mk); Mesut Pinar (Mp); Muzaffer Mükemre (Mm); M.Tekşen (Mt); Nasip Demirkuş (Nd); Murat Ünal (Mü);
Tuncay Dirmenci (Td); Osman Kara Karabacak (Ok); Ömer Bingöl (Öb); Turan Çelik (Tç).

IUCN Red Data categories were cited with the following abbreviations: Critically endangered (cr); Data deficient (dd); Endangered (en); Extinct (ex); Extinct in the wild (ew); Lower risk (lr); Least concern (lc); Conservation dependent (cd); Near threatened (nt); Not evaluated (ne); Vulnerable (vu) (Ekim et al. 2000). The taxa collected by the authors are marked with an asterisk $(*)$.

Table 1. Collection localities of the endemic within Van Lake basin.

\begin{tabular}{|l|l|c|}
\hline No & Locality & Altitude \\
\hline 1 & B9 Van: Değirmen Village, Erek Mountain & $2000-2500 \mathrm{~m}$ \\
\hline 2 & B9 Van: Değirmen Village, Erek Mountain & $2000-2300 \mathrm{~m}$ \\
\hline 3 & B9 Van: Gürpınar, Kurubaş pass & $1900 \mathrm{~m}$ \\
\hline 4 & B9 Van: Hoşap, Güzeldere pass, around guardhouse & $2600-2730 \mathrm{~m}$ \\
\hline 5 & B9 Van: Tevekli and Alaköy meadows & $1850 \mathrm{~m}$ \\
\hline 6 & B9 Van: Hoşap, Güzeldere pass from fountain to gourdhouse & $2600-2730 \mathrm{~m}$ \\
\hline 7 & B9 Van: Van to Erciş 35. Km & $1850 \mathrm{~m}$ \\
\hline 8 & B9 Van: Hoşap, Güzeldere pass & $2250 \mathrm{~m}$ \\
\hline 9 & B9 Van: Hoşap, Güzeldere pass & $2500 \mathrm{~m}$ \\
\hline
\end{tabular}




\begin{tabular}{|c|c|c|}
\hline 10 & B9 Van: Hoşap, Güzeldere pass & $2750 \mathrm{~m}$ \\
\hline 11 & B9 Van: Gevaş, Artos Mountain, west slopes & $2000-2350 \mathrm{~m}$ \\
\hline 12 & B9 Van: Artos Mountain, north side & $2200-2500 \mathrm{~m}$ \\
\hline 13 & B9 Van: Hoşap, Güzeldere pass & $2600-2730 \mathrm{~m}$ \\
\hline 14 & B9 Van: From Van to Çatak road $70 . \mathrm{km}$ & $1750-2000 \mathrm{~m}$ \\
\hline 15 & B9 Van: from Gürpınar to Çatak $35 . \mathrm{km}$ & $2200-2500 \mathrm{~m}$ \\
\hline 16 & B9 Van: Çaldıran, Tendürek Mountains & $2650 \mathrm{~m}$ \\
\hline 17 & B9 Van: Erek Mountain meadows of Değirmen Village & $2600 \mathrm{~m}$ \\
\hline 18 & B9 Van: Değirmen Village, Erek Mountain & $2400 \mathrm{~m}$ \\
\hline 19 & B9 Van: Muradiye, meadows of Yumaklı Village & $2250 \mathrm{~m}$ \\
\hline 20 & B9 Van: Hoşap, Güzeldere pass, around FedaiTaşı & $2780-2900 \mathrm{~m}$ \\
\hline 21 & B9 Van: Hoşap, Güzeldere pass & $2650 \mathrm{~m}$ \\
\hline 22 & B9 Van: Bahçesaray, from Müküs Water Cavern to Sündüs plateau & $1700-2000 \mathrm{~m}$ \\
\hline 23 & B9 Van: Erek Mountain, Şuşanıs Village steppe slopes & $2300 \mathrm{~m}$ \\
\hline 24 & B9 Van: Hoşap, Güzeldere pass & $2400-2700 \mathrm{~m}$ \\
\hline 25 & B9 Van: Erek Mountain, Şuşanıs Village slopes & $2400-2500 \mathrm{~m}$ \\
\hline 26 & B9 Van: Around Ayanıs Villages & $1750 \mathrm{~m}$ \\
\hline 27 & B9 Van: Gürpınar, Kurubaș pass & $2000 \mathrm{~m}$ \\
\hline 28 & B9 Van: Zernek Dam $(60-62 . \mathrm{Km})$ north slopes & $2000 \mathrm{~m}$ \\
\hline 29 & B9 Van: Hoşap, Güzeldere pass, around highway station & $2750 \mathrm{~m}$ \\
\hline 30 & B9 Van: Gürpınar, Kurubaş pass & $1800-2000 \mathrm{~m}$ \\
\hline 31 & B9 Van: Hoşap, Güzeldere pass, around sentry & $2750 \mathrm{~m}$ \\
\hline 32 & B9 Van: Hoşap, Güzeldere pass, around highway station & $2790 \mathrm{~m}$ \\
\hline 33 & B9 Van: Özalp road $41 . \mathrm{Km}$ & $1970 \mathrm{~m}$ \\
\hline 34 & B9 Van: Kopanis Village, Erek Mountain & $2150-2700 \mathrm{~m}$ \\
\hline 35 & B9 Van: Kopannis Village, Erek Mountain & $2500-2700 \mathrm{~m}$ \\
\hline 36 & B9 Van: Özalp road 20. Km, slopes & $2000 \mathrm{~m}$ \\
\hline 37 & B9 Van: Hoşap, Güzeldere pass, around fountain & $2500-2650 \mathrm{~m}$ \\
\hline 38 & B9 Van: Hoşap, Güzeldere pass, around FedaiTaş1 & $2750-2900 \mathrm{~m}$ \\
\hline 39 & B9 Van: Bahçesaray, Sündüz plateau, valley slopes & $2100 \mathrm{~m}$ \\
\hline 40 & B9 Van: Bahçesaray, from Krapet pass to Liçan Village & $2700 \mathrm{~m}$ \\
\hline 41 & B9 Van: Hoşap, Güzeldere pass, around sentry, & $2750-2800 \mathrm{~m}$ \\
\hline 42 & B9 Van: Bahçesaray, around Liçan Village & $1900-2000 \mathrm{~m}$ \\
\hline 43 & B9 Van: Muradiye, Suphan Lake, meadows of Adaklı Village & $2350-2500 \mathrm{~m}$ \\
\hline 44 & B9 Van: Around Muradiye Waterfall & $2000 \mathrm{~m}$ \\
\hline 45 & B9 Van: Muradiye, $3 \mathrm{Km}$ to Çaldıran & $2150 \mathrm{~m}$ \\
\hline 46 & B9 Van: Muradiye $2 \mathrm{Km}$ to Çaldıran, Ayrancılar Village & $2150-2200 \mathrm{~m}$ \\
\hline 47 & B9 Van: Hoşap, Güzeldere pass & $2500-2750 \mathrm{~m}$ \\
\hline 48 & B9 Van: Cross of Çatak and Bahçesaray roads & $2000 \mathrm{~m}$ \\
\hline 49 & B9 Van: From Gürpınar to Çatak, before Görentaş Village & $2000 \mathrm{~m}$ \\
\hline 50 & B9 Van: Bahçesaray, from krapet pass to Liçan Village & $2500-2800 \mathrm{~m}$ \\
\hline 51 & B9 Van: Bahçesaray, around Müküs Water Cavern & $1800-1850 \mathrm{~m}$ \\
\hline 52 & B9 Van: Bahçesaray, from Liçan Village to Krapet pass & $1800-2500 \mathrm{~m}$ \\
\hline 53 & B9 Van: Kopannis Village, Erek Mountain & $3000-3200 \mathrm{~m}$ \\
\hline 54 & B9 Van: Kopannis Village, Erek Mountain & $2500-3000 \mathrm{~m}$ \\
\hline 55 & B9 Van: from Gürpınar to Hoşap 10. Km, around road & $1900 \mathrm{~m}$ \\
\hline 56 & B9 Van: from Van to Tatvan & $2500 \mathrm{~m}$ \\
\hline 57 & B9 Van: Hoşap, Güzeldere pass, around fountain & $2650 \mathrm{~m}$ \\
\hline 58 & B9 Van:Erciş District, northeast of Aksakal village, Kırdekar hill & $2692 \mathrm{~m}$ \\
\hline 59 & C9 Van: Çatak, Konalga village, Tanrıverdi hamlet, around Zevviçal & $2372 \mathrm{~m}$ \\
\hline 60 & B9 Van: Miks (Bahçesaray) district, Çıravis Mountains (Çiyayê Çirawîs) & $3343 \mathrm{~m}$ \\
\hline 61 & B9 Van: Ahta Dağ, southeast of Kalecik village near Özalp & $2500-2700 \mathrm{~m}$ \\
\hline 62 & B9 Van: Bahçesaray, Karabel (Kirapit) pass & $3200-3400 \mathrm{~m}$ \\
\hline 63 & B9 Van: Çatak, North of Çatak Valley, surroundings of Bilgi Village & $1723 \mathrm{~m}$ \\
\hline 64 & B9 Bitlis: Adilcevaz, Suphan Mountain north of Kıçkılı village & $2500 \mathrm{~m}$ \\
\hline 65 & B9 Van: ,Bahçesaray, from Altındere village to Kavushhahap Mount. & $2600 \mathrm{~m}$ \\
\hline 66 & B9 Van, Bahçesaray, Serkani location & $1800 \mathrm{~m}$ \\
\hline 67 & B9 Van: Bahçearay, Arnos Mountain, & $2200 \mathrm{~m}$ \\
\hline 68 & B9 Van: between Çatak and Bahçesaray, north of Yukarınarlı village & $2200-2400 \mathrm{~m}$ \\
\hline 69 & B9 Van: between Başkale and Van & $2052 \mathrm{~m}$ \\
\hline 70 & B9 Van: Gevaş, Deveboynu peninsula, above İnköy & $2100 \mathrm{~m}$ \\
\hline 71 & B9 Van: Başkale, Çaldıran village & $2000-2050 \mathrm{~m}$ \\
\hline 72 & B9 Van: Muradiye above the Adakl1, village & $2300 \mathrm{~m}$ \\
\hline 73 & B9 Van: from Van to Muradiye, Bendi mahi region & $1659 \mathrm{~m}$ \\
\hline 74 & B9 Van: Çatak, Kavuşşahap Mountain, Karapet pass & $2750 \mathrm{~m}$ \\
\hline 75 & B10 Van:Van- Hakkari road, around Başkale & $2100 \mathrm{~m}$ \\
\hline 76 & B9 Van: Gevaş, Artos Mountain & $2500 \mathrm{~m}$ \\
\hline
\end{tabular}




\begin{tabular}{|c|c|c|}
\hline 77 & B9 Van: Gürpınar-Başkale road, around Hoşap (Güzelsu) & $2050 \mathrm{~m}$ \\
\hline 78 & B9 Van: Hoşab (Güzeldere) province, Güzeldere passage & $2791 \mathrm{~m}$ \\
\hline 79 & B9 Van: from Narlıca Village to Bahçesaray, Karapetpass & $2885 \mathrm{~m}$ \\
\hline 80 & B9 Van: between Van-Başkale, Güzeldere pass & $2738 \mathrm{~m}$ \\
\hline 81 & B9 Van: Gürpınar, Iş1kpınar to Hacıköy, around the pond & $2154 \mathrm{~m}$ \\
\hline 82 & B9 Van: From Vari Krapet pass to Bahçesaray & $2500-2700 \mathrm{~m}$ \\
\hline 83 & B9 Van: Gürpınar, behind Koçgüden village & $2500 \mathrm{~m}$ \\
\hline 84 & B9 Van: Erciş, 1. Km to the Yukarıgöze neighborhood & $2000-2200 \mathrm{~m}$ \\
\hline 85 & B9 Van: Gürpınar, Hamurkesen Village, inside the valley & $2400 \mathrm{~m}$ \\
\hline 86 & B10 Van: Özalp, Aşağı Koçkıran Village, towards Karatepe & $2275 \mathrm{~m}$ \\
\hline 87 & C9 Van: Gürpınar, from Üçdoğan to Bükülmez Village & $2153 \mathrm{~m}$ \\
\hline 88 & B9 Van: Erciş, Taşkapı village, Karakaya & $2448 \mathrm{~m}$ \\
\hline 89 & B9 Van: Gürpınar, in the west of Tutmaç village & $2800 \mathrm{~m}$ \\
\hline 90 & B9 Van: Gürpınar, the opposite of Koçgüden village & $2300 \mathrm{~m}$ \\
\hline 91 & B9 Van: Özalp, East of Kırkçalı & $1900-2050 \mathrm{~m}$ \\
\hline 92 & B9 Van: Muradiye, Kemerköprü Village, Akçadağ, Neri Tepe & $2000 \mathrm{~m}$ \\
\hline 93 & B9 Van: Özalp, from Y. Ayazca to Y. Mollahasan village & $2003 \mathrm{~m}$ \\
\hline 94 & B10 Van: Özalp, from Așağı Tulgalı to Y.Dönerdere Village & $2053 \mathrm{~m}$ \\
\hline 95 & B9 Van: Gürpınar, behind the Koçgüden village & $2950 \mathrm{~m}$ \\
\hline 96 & B9 Van: Çatak, from Çatak to the Kirazlı village, south slope & $1800 \mathrm{~m}$ \\
\hline 97 & C9 Van: Gürpınar, from Topçudeğirmeni to Yalınca Village & $2269 \mathrm{~m}$ \\
\hline 98 & B9 Van: Gürpınarthe behind of Tutmak village & $2600 \mathrm{~m}$ \\
\hline 99 & B9 Van: Gürpınar, Kapçık village, the edge of the creek & $2134 \mathrm{~m}$ \\
\hline 100 & B9 Van: Özalp, A.Sağmallı village, Pirreşit Mountain & $2500 \mathrm{~m}$ \\
\hline 101 & B9 Van: Muradiye, Görecek village, around Şevlii & $2200-2500 \mathrm{~m}$ \\
\hline 102 & B9 Van: Özalp, from Y.Koçkıran to A.Koçkıran village & $2300 \mathrm{~m}$ \\
\hline 103 & B9 Van: Özalp, A.Akçagöl village "Pirreșit" mountain & $2293 \mathrm{~m}$ \\
\hline 104 & B10 Van: Özalp, Y.Balçıklı village plateau & $2300 \mathrm{~m}$ \\
\hline 105 & B9 Van: Erçek, Erçek Lake shores & $1800 \mathrm{~m}$ \\
\hline 106 & B9 Van: Muradiye, Babacan village & $1900 \mathrm{~m}$ \\
\hline 107 & B9 Van: Erciş, around of Soluhan village & $2634 \mathrm{~m}$ \\
\hline 108 & B9 Van: Erciş, around of Şehirpazarı village & $1937 \mathrm{~m}$ \\
\hline 109 & B9 Van: Gürpınar, from Taşdöndüren village to Belkış slope & $2300 \mathrm{~m}$ \\
\hline 110 & B9 Bitlis: Tatvan, Tanriyer village & $1700 \mathrm{~m}$ \\
\hline 111 & B9 Van: Erciş, from Doluca to Gürgürbaba hill & $2912 \mathrm{~m}$ \\
\hline 112 & B9 Van: Gürpınar, from Hamurkesen to Işıkpınar village & $2000 \mathrm{~m}$ \\
\hline 113 & B9 Bitlis: Hizan, from Hizan to Sürmecek village & $1500 \mathrm{~m}$ \\
\hline 114 & B9 Van: Muradiye, Görecek village, around Şevkii & $2350 \mathrm{~m}$ \\
\hline 115 & B9 Van: Gürpınar, from Sarıyaprak to Güleçler village & $2300 \mathrm{~m}$ \\
\hline 116 & B9 Van: Gürpınar, from Cevizalan to Umut Village & $2500 \mathrm{~m}$ \\
\hline 117 & B9 Van: Çatak, Bilgi village, south slopes & $1900 \mathrm{~m}$ \\
\hline 118 & B9 Van: Erciş, from the village of Taşkap1 to the ganissipi & $2200 \mathrm{~m}$ \\
\hline 119 & B9 Van: Erciş, Şehirpazarı village, Adalar plateau & $1937 \mathrm{~m}$ \\
\hline 120 & B9 Van: Erciş, Şehirpazarı village, Adalar plateau, behind the spa & $1950 \mathrm{~m}$ \\
\hline 121 & B9 Van: Özalp, Sugeçer and Boğazkesen villages, Ziyaret hill & $2400 \mathrm{~m}$ \\
\hline 122 & B9 Van: Erciş, around Şehirpazarı village & $2293 \mathrm{~m}$ \\
\hline 123 & B9 Van: Erciş, Altındere village, hara surroundings & $1845 \mathrm{~m}$ \\
\hline 124 & B9 Van: Özalp, from Y.Ayazca to Mollahasan village & $2003 \mathrm{~m}$ \\
\hline 125 & B9 Bitlis: around İçmeli village, & $1510 \mathrm{~m}$ \\
\hline 126 & B9 Van: Muradiye Kemerköprü village, around Akçadağ & $2200 \mathrm{~m}$ \\
\hline 127 & B9 Van: Erciş, Şehirpazarı village, Adalar plateau & $1950 \mathrm{~m}$ \\
\hline 128 & B9 Van: Özalp, south of Y. Çavdarlık village & $2219 \mathrm{~m}$ \\
\hline 129 & B9 Van: Gürpınar, from Hoşap to Zernek barrage, stream edge & $1900 \mathrm{~m}$ \\
\hline 130 & B9 Van: Hoşap, Güzeldere pess & $2400 \mathrm{~m}$ \\
\hline 131 & B9 Van: Özalp Ahta Mountain & $2700 \mathrm{~m}$ \\
\hline 132 & B9 Van: Gürpınar, Geçerli village & $1890 \mathrm{~m}$ \\
\hline 133 & B9 Van: Gürpınar, north of Yedisalkım village & $2400 \mathrm{~m}$ \\
\hline 134 & B9 Van: Kurubaş pass, Doğanlar village & $1900 \mathrm{~m}$ \\
\hline 135 & B9 Van:Gürpınar, Taş̧döndüren village roadsides & $1900 \mathrm{~m}$ \\
\hline 136 & B9 Van:Gürpınar, Taşdöndüren village roadsides & $1900 \mathrm{~m}$ \\
\hline 137 & B9 Van: Erciş, around Ilıca & $1912 \mathrm{~m}$ \\
\hline 138 & B9 Bitlis: Hizan, Sarpkaya village & $2000 \mathrm{~m}$ \\
\hline 139 & B9 Van: Gürpınar, from Ișıkpınarı village to Südis Mountain & $2350 \mathrm{~m}$ \\
\hline 140 & B9 Van: from Dorutay to Aksaağaç and Yumruklu villages & $2100 \mathrm{~m}$ \\
\hline 141 & B9 Van: Erciş, from Taşkapı village to Hüdavendigar Mountain & $3186 \mathrm{~m}$ \\
\hline 142 & B10 Van: Başkale,Darıca village road, steppe around the bridge & $1920 \mathrm{~m}$ \\
\hline 143 & B9 Van: Gürpınar, above the Kochgüden village & $2800 \mathrm{~m}$ \\
\hline
\end{tabular}




\begin{tabular}{|c|c|c|}
\hline 144 & B9 Bitlis: Adilcevaz, the north of the Kıçıklı village & $2400-2700 \mathrm{~m}$ \\
\hline 145 & B9 Van: Muradiye, Derviş Plateau, Hacı Cave, Kom stream & $2000-2200 \mathrm{~m}$ \\
\hline 146 & B9 Van: Gürpınar, Sarıyaprak Plateau-Güleçler village & $2400 \mathrm{~m}$ \\
\hline 147 & B9 Van: Özalp, Mollahasan village & $2380 \mathrm{~m}$ \\
\hline 148 & B9 Van: Gürpınar, behind the Hamurkesen village & $2300 \mathrm{~m}$ \\
\hline 149 & B9 Van: Gürpınar, southeastern slopes of the Başet mountain & $2400 \mathrm{~m}$ \\
\hline 150 & B9 Van: Erciş, around Doluca village & $1965 \mathrm{~m}$ \\
\hline 151 & B10 Van: Başkale, around Oğulveren village & $2250 \mathrm{~m}$ \\
\hline 152 & B9 Van: Gürpınar, from Bölmeçalı to Hacıköy village & $2500 \mathrm{~m}$ \\
\hline 153 & B9 Van: Erciş, the edge of Zilan Creek & $1700 \mathrm{~m}$ \\
\hline 154 & B10 Van: Özalp, around Özalp & $2300 \mathrm{~m}$ \\
\hline 155 & B9 Van: Özalp, Kaşıkara village surroundings & $1800 \mathrm{~m}$ \\
\hline 156 & B9 Van: Muradiye, Beşparmak village surroundings & $2300 \mathrm{~m}$ \\
\hline 157 & B9 Bitlis: Tatvan, Kesan brook 4.km & $1700 \mathrm{~m}$ \\
\hline 158 & B9 Van: Özalp, in the West of Şehittepe village & $2435 \mathrm{~m}$ \\
\hline 159 & B10 Van: Özalp, Yukaru Tulgalı village, Kel hill & $2280 \mathrm{~m}$ \\
\hline 160 & B9 Van: Özalp, from Bodurağaç to Seydibey village & $2194 \mathrm{~m}$ \\
\hline 161 & B9 Van: Gürpınar, from Hamurkesen to Işıkpınar village & $2000 \mathrm{~m}$ \\
\hline 162 & B9 Van: Erciş, from Taşkapı village to Hüdavendigar Mountain & $2100-2800 \mathrm{~m}$ \\
\hline 163 & B9 Van: Erciş, the edge of Zilan creek & $1750 \mathrm{~m}$ \\
\hline 164 & B9 Van: Erçek Lake Coast & $1800 \mathrm{~m}$ \\
\hline 165 & B9 Van: Muradiye, south slopes of the Pirreşit Mountain & $2000 \mathrm{~m}$ \\
\hline 166 & B9 Van: Gürpınar, the back side of Yedisalkım village & $2200 \mathrm{~m}$ \\
\hline 167 & B9 Van: Gürpınar, south of Hamurkesen village & $1900 \mathrm{~m}$ \\
\hline 168 & B9 Van: Erciss, Doluca village, Kuzubulak lake periphery & $2535 \mathrm{~m}$ \\
\hline 169 & B9 Van: Özalp, east of Seydibey village & $2100 \mathrm{~m}$ \\
\hline 170 & B9 Van: Gürpınar, the southern slopes of Başet Mountain & $2600 \mathrm{~m}$ \\
\hline 171 & B9 Van: Muradiye, Görecek village, Şevki location & $2200-2500 \mathrm{~m}$ \\
\hline 172 & B9 Van: Özalp, from A.Molahasan to Y.Molahasan village & $1800 \mathrm{~m}$ \\
\hline 173 & B9 Van: Gürpınar, Zernek barrage, roadside & $1850 \mathrm{~m}$ \\
\hline 174 & B9 Van: Erciş, Sabanbüken village, Çakali plateau & $2825 \mathrm{~m}$ \\
\hline 175 & B9 Bitlis: Tatvan, at the 7th km of Kesan stream & $1700-1800 \mathrm{~m}$ \\
\hline 176 & B9 Van: Gürpınar north slopes of Zernek Dam & $1900 \mathrm{~m}$ \\
\hline 177 & B9 Van: Gürpınar, around Yurtbașı village & $2100 \mathrm{~m}$ \\
\hline 178 & B9 Bitlis: Adilcevaz, from Çanak yayla to Cihangir village, & $2300-2500 \mathrm{~m}$ \\
\hline 179 & B9 Van : Gürpınar, northwest of Koçgüden village & $2950 \mathrm{~m}$ \\
\hline 180 & B9 Van: Erciş, Dolucan vilage, Lake Şama, Lignite man. periphery & $2634 \mathrm{~m}$ \\
\hline 181 & B9 Van: Erciş, Altındere stud farm circumference & $1845 \mathrm{~m}$ \\
\hline 182 & B9 Van: Özalp, Kalecik village & $2000 \mathrm{~m}$ \\
\hline 183 & B9 Van: Muradiye, Görecek village, Şevki location & $2200-2500 \mathrm{~m}$ \\
\hline 184 & B9 Van: Van castle, rock crevices & $1720 \mathrm{~m}$ \\
\hline 185 & B9 Van: Erciş, Doluca village, Gürgürbaba hill & $2738 \mathrm{~m}$ \\
\hline 186 & B9 Van: Muradiye, slopes of Pirreșit Mountain opposite Özalp & $2000 \mathrm{~m}$ \\
\hline 187 & B9 Van: Erciş, around the lake İkizçalı & $1978 \mathrm{~m}$ \\
\hline 188 & B9 Bitlis: Tatvan, the Kesan brook $4 . \mathrm{km}$ & $1700 \mathrm{~m}$ \\
\hline 189 & B9 Van: Özalp, the south of Y. Koçkıran village & $2050 \mathrm{~m}$ \\
\hline 190 & B9 Bitlis: Adilcevaz, from Harmantepe to Çanakyayla village & $2200 \mathrm{~m}$ \\
\hline 191 & B9 Van: Özalp, the north of Y. Yargılı village, Pirreşit Mountain & $2070 \mathrm{~m}$ \\
\hline 192 & B9 Van: the west of Erek Mountain & $2500 \mathrm{~m}$ \\
\hline 193 & B9 Van: Özalp, from Özalp to Dorutay village, Koyun yatağ1 & $2072 \mathrm{~m}$ \\
\hline 194 & B9 Van: Gürpınar, opposite of Koçgüden village & $2500 \mathrm{~m}$ \\
\hline 195 & B9 Van: Gürpınar, from Işıkpınar village to Hacı village & $2100 \mathrm{~m}$ \\
\hline 196 & B9 Van: Erciş, around Ulupamir village, creek edge & $1811 \mathrm{~m}$ \\
\hline 197 & B9 Van: Özalp, Damlacik village, wheat field & $2300 \mathrm{~m}$ \\
\hline 198 & B9 Van: Muradiye, the edges of the valley behind Adaklı village & $2400 \mathrm{~m}$ \\
\hline 199 & B9 Bitlis: Adilcevaz, the north of Bahçedere village & $2100 \mathrm{~m}$ \\
\hline 200 & B9 Van: Özalp, Yukarı Tulgalı village, border plateau & $2250 \mathrm{~m}$ \\
\hline 201 & B9 Van: Gürpınar, Sarıyaprak plateau, valley inside & $2800 \mathrm{~m}$ \\
\hline 202 & B9 Van: Muradiye, along the Yumaklı valley & $2200 \mathrm{~m}$ \\
\hline 203 & B9 Van: Erciş, the northwestern part of Ulupamir village & $1871 \mathrm{~m}$ \\
\hline 204 & B9 Van: Gürpınar, from Zernek Dam to Üçgen vilage & $1700-1900 \mathrm{~m}$ \\
\hline 205 & B10 Van: Özalp, from A. Koçkıran vilage to Iranian borderı & $2200 \mathrm{~m}$ \\
\hline 206 & B9 Bitlis: Tatvan, Kesan creek 10-15.km, road side & $1550 \mathrm{~m}$ \\
\hline 207 & B9 Van: Muradiye, Ünseli village, Akçadağ (Esruk) mountain & $2400 \mathrm{~m}$ \\
\hline 208 & B10 Van: Başkale, Esenyamaç village $3 . \mathrm{km}$ north slope & $1976 \mathrm{~m}$ \\
\hline 209 & B9 Van: Erciş, from Ilıca to Yeknal plateau & $2019 \mathrm{~m}$ \\
\hline 210 & B9 Van: Muradiye, $3 \mathrm{~km}$ to the village of Görecek & $1800 \mathrm{~m}$ \\
\hline
\end{tabular}




\section{FAM: RANUNCULACEAE}

*Delphinium carduchorum Chowdhuri \& P.H.Davis: meadows, locality 29; 19.07.1997, Nd 6191 -Mk 12755; meadows, locality 47; 13.07.1997, Nd 6179, 6191Mk 12743, 12755; rocky slopes, locality 54; 27.07.1997, Nd 6225 -Mk 12789;locality 56; 23.07.1997, Nd 6304a Mk 12867a, IUCN: nt.

*Delphinium dolichostachium Chowdhuri \& P.H.Davis: road slopes, steppe, locality 52; 16.07.1997, Nd 5945-Mk 12509, IUCN: cd.

*Delphinium cyphoplectrum Boiss. var. vanense (Rech. Fil) P.H. Davis: rocky slopes, locality 48 ; 15.07.1997, Nd 5883-Mk 12447; meadows, locality 47; 13.07.1997, Nd 6189-Mk 12753; rocky slopes, locality 54; 27.07.1997, Nd 6238 -Mk 12802; steppe, locality 57; 19.07.1997, Nd 6209 -Mk 12773, IUCN: cd.

Delphinium cyphoplectrum Boiss. var. stenophyllum Boiss.: steppe, locality 83; 31.07.2002, Mü 7800.

*Ranunculus crateris P.H. Davis : meadows, locality 17; 08.06.1997, Mk 11836-Nd 5266, IUCN: cd. Variations: Stems up to 12 flowered (stems 1-6 flowered), all sepals not reflexed (sepals reflexed).

*Ranunculus fenzlii Boiss.: steppe, locality 21; 13.06.1997, Mk 11901, 11922 -Nd 5331, 5352, IUCN: lc.

Ranunculus bingoeldaghensis A.Engin: locality 84; 19.05.2002, Ok 2931 IUCN: en.

*Ranunculus vanensis P.H. Davis: B9 Van: steppe, locality 38; 27.06.1997, Mk 12260-Nd 5688, IUCN: cd. Variations: Early stage of upper leaves linear lanceolate, later divided to linear lobes, some samples include undivided and divided upper leaves. Early stage of achene semicircular, later semicircular to slender. Posterior of achene beak brownish to blackish (unknown).

*Ranunculus munzurensis S.Erik \& Yildirimli: Rocky slopes, locality 12; 26.05.1997, Nd 5211 -Mk 11747, IUCN: cd.

\section{FAM: PAPAVERACEAE}

Papaver persicum Lindl. subsp. fulvum Kit-Tan \& Sorger: steppe, locality 85; 23.07.2003, Mü 8832, IUCN: lc.

*Papaver fugax Poiret var. platydiscus Cullen: steppe, locality $31 ; 21.06 .1997$, Mk 12053-Nd 5480; steppe locality 38; 27.06.1997, Mk 12224 - Nd 5652, IUCN: lc.

\section{FAM: BRASSICACEAE}

Isatis cappadocica Desv. subsp. alyssifolia (Boiss.) P.H. Davis: locality 86; 05.07.1997, Fö 4767, IUCN: nt.

Isatis erzurumica P.H.Davis: creek edge, locality 87; 16.06.2007, Mf 1856, IUCN: nt.

*Isatis candolleana Boiss.: $\quad$ steppe, locality 41; 19.07.1997, Nd 6100, IUCN: lc.

*Isatis undulata Aucher ex Boiss.: wet meadows, locality 35; 22.06.1997, Mk 12157-Nd5585, IUCN: en.

*Isatis aucheri Boiss : rocky slopes, locality 34; 22.06.1997, Mk 12114, 12159 -Nd5542, 5587; meadows, locality 43; 12.07.1997, Nd 5792 -Mk 12353, IUCN: lc.
*Isatis glauca Aucher\&Boiss.

subsp. icaonia (Boiss. \& Heldr.) P.H. Davis: rocky slopes, locality 26; 19.06.1997, Mk 11974 - Nd 5403; slopes, locality 36; 26.06.1997, Mk 12212 - Nd 5640, IUCN: lc.

Isatis spatella P.H. Davis : sandy soil area, locality 39; 02.05.1999, Mf; 1013, IUCN: en.

Physocardamum davisii Hedge: steppe, calcareous soil, locality 88; 21.06.2007, Ok 6558, IUCN: vu.

Aethionema froedinii Rech. fil.: road slopes, locality 50; 15.07.1997, Mf 1162. IUCN: lc.

*Aethionema eunomioides (Boiss.) Bornm.: rocky slopes, locality 53; 16.07.1997, Nd 7016, IUCN: lc.

Aethionema iberideum (Boiss.) Boiss : steppe area, locality 89; 08.06.2003, Mü 8358.

Aethionema caespitosum (Boiss.) Boiss.: steppe area, locality 89; 08.06.2003, Mü 8359., IUCN: nt.

*Bornmuellera cappadocica (DC.) Cullen \& Dudley: steppe, locality 38; 27.06.1997, Mk 12234-Nd 5662, IUCN: lc.

Alyssum huetii Boiss.: steppe, locality 90; 27.05.2001, Mü4935b, IUCN: lc.

Alyssum blepharocarpum Dudley\&Hub.-Mor.: locality 91; 27.06.1996, Fö 2530, IUCN: nt.

Alyssum ochroleucum Boiss.: steppe, locality 92; 21.06.2001, Ok 1859, IUCN: lc.

Alyssum aurantiacum Boiss.: locality 93; 02.07.1997, F 5155, IUCN: cd.

Alyssum niveum Dudley: steppe, locality 94; 10.07.1997, F 4916, IUCN: en.

Alyssum pateri Nyár. subsp. prostratum (Nyár.) Dudley: steppe, locality 95; 31.07.2002, Mü 7798, IUCN: lc.

Alyssum filiforme Nyár.: steppe, locality 96; 21.06.2003, Mp 1973, IUCN: lc.

Alyssum haussknechtii Boiss.: steppe, locality 97; 26.07.2006, Mf 392, IUCN: Lr (cd)

*Draba rosularis Boiss.: steppe, locality 10; 24.05.1997, Mk11697-Nd 5163; steppe, locality 21; 13.06.1997, Mk 11906- Nd 5336, IUCN: lc.

*Draba cappadocica Boiss. \& Bal.: steppe, locality 38; 27.06.1997, Mk 12226- Nd 5654, IUCN: lc.

Draba orientalis Karabacak \& Behçet: Holotype, rocky crevices, locality 58; 28.05.2007, Ok 6081.

Arabis carduchorum Boiss.: steppe, locality 98; 23.07.2002, Mü 7563, IUCN: nt.

Sterigmostemum sulphureum Bornm. subsp. glandulosum Hub.-Mor. \& Reese: the edge of the creek, locality 99; 16.06.2007, Mf 1721, IUCN: vu.

Erysimum sintenisianum Bornm.: steppe, locality 100; 26.06.1997, Fö 3513, IUCN: lc.

Erysimum echinellum Hand.-Mazz.: steppe, locality 142; 31.05.2008, Da 1246b, IUCN: en. 


\section{FAM: CARYOPHYLLACEAE}

*Minuartia umbellifera (Boiss.) Mc Neill subsp. umbellifera var. kurdica McNeil: meadows, locality 29; 19.07.1997, Nd 6114 -Mk 12676; rocky slopes, locality 53; 16.07.1997, Nd 5951, 5973, 6001- Mk 12515, 12535, 12564, IUCN: nt.

Minuartia anatolica (Boiss.) Woronow var. anatolica: steppe, locality 143; 16.07.2002, Mü7349, IUCN: cd.

*Minuartia glandulosa (Boiss. \& Huet) Bornm.: rocky slopes, locality 54; 27.07.1997, Nd 6232 -Mk 12796, IUCN: lc.

*Cerastium araraticum Rupr.: rocky slopes, locality 53; 16.07.1997, Nd 5968 -Mk12531, IUCN: lc.

*Cerastium gnaphalodes Fenzl : rocky slopes, locality 53; 16.07.1997, Nd 5967, IUCN: 1c.

*Dianthus lactiflorus Fenzl : rocky slopes, locality 53; 16.07.1997, Nd 5966, IUCN: cd.

*Dianthus erythrocoleus Boiss.: meadows locality 29; 19.07.1997, Nd 6204- Mk 12768, Nd 6059, 6101- Mk 12622, 12663; meadows , locality 46; 13.07.1997, Nd 5834 -Mk 12395; meadows, locality 47; 13.07.1997, Nd 6133 -Mk 12695; rocky slopes, locality 53; 16.07.1997,Nd 5970, 6022- Mk 12533, 12585; rocky slopes, locality 54; 27.07.1997, Nd 5765 -Mk 12327, IUCN: lc.

*Dianthus masmaneus Boiss. var. glabrescens Boiss. : meadows, locality 43;12.07.1997, Nd 5804 -Mk 12365, IUCN: 1c.

Dianthus muschianus Kotschy \& Boiss.: wet meadows, locality 24: 23.07.1997, Mf 1835, Nd 6884z, IUCN: lc.

*Dianthus zederbaueri Vierh.: meadows, locality 43; 12.07. 1997, Nd 5803, IUCN: cd.

Dianthus vanensis Behçet \& İlçim: , steppe, locality 59; 25.06.2010, Mm 300.

*Gypsophila adenophylla Bark.: slopes, steppe, locality 52; 16.07.1997, Nd 5971 -Mk 12534, IUCN: lc.

*Gypsophila bitlisensis Bark.: steppe slopes, locality 27; 21.06.1997, Mk 12023-Nd 5451, IUCN: cd.

*Silene capitellata Boiss.: meadows, locality 15; 05.06.1997, Mk 11755, IUCN: lc.

*Silene muradica Schschkin.: steppe, locality 44; 13.07.1997, Nd 5811 -Mk 12372, IUCN: lc.

Silene cartilaginea Hub.-Mor.: steppe, locality 30; 13.06.1999, Ma 1603, IUCN: cd.

*Silene lucida Chowdh. subsp. lucida: steppe, locality 38; 27.06.1997, Nd 5680, 6799, IUCN: lc.

*Silene sclerophylla Chowdh.: $\quad$ steppe, locality 30; 13.06.1999, Nd 6598d, IUCN: 1c.

Silene miksensis F1rat \& K.Yıld1z: limestone and rocky slopes, locality 60; 10.08.2011, Mf 27345.

Silene acaulis (L.) Jacq. subsp. vanensis Özgökçe \& Kit Tan: locality 61; 15.05.1998, Fö 5911

\section{FAM: POLYGONACEAE}

Rumex ponticus E.H. L. Krause: steppe rocky place, locality 101; 08.07.2001, Ok 2076. IUCN: lc.

\section{FAM: HYPERICACEAE}

Hypericum thymbrifolium Boiss. \& Noë: steppe, locality 102; 04.07.1997, Fö 4610, IUCN: cd.

\section{FAM: LINACEAE}

Linum unguiculatum Da P.H. Davis vis: steppe, locality 103; 26.06.1997, Fö 3407. IUCN: lc.

Linum obtusatum (Boiss.) Stapf: steppe, locality 104; 23.5.1998, Fö 5946, IUCN: 1c.

\section{FAM: GERANIACEAE}

Geranium ibericum Cav. subsp. jubatum (Hand.-Mazz.) Da P.H. Davis: damp rocky, locality 144; 08.07.2001, B 6648, IUCN: 1c.

Erodium hakkiaricum P.H.Davis: steppe, locality 146; 18.07.2001, Mü 6068, IUCN: en.

Erodium amanum Boiss. \& Kotschy: locality 144; 09.07.1987, B 346, IUCN: 1c.

\section{FAM: RUTACEAE}

Haplophyllum cappadocicum Spach: steppe, locality 147; 30.06.1998, Fö 8168, IUCN: nt.

\section{FAM: FABACEAE}

Astragalus hirsutus Vahl: rocky-steppe, locality 148; 22.05.2002, Mü 6805, IUCN: lc.

Astragalus ovatus DC.: steppe, locality 149; Mü 8503, 10.07.2003, IUCN: dd.

*Astragalus dasycarpus Chamberlain: meadows, locality 29; 19.07.1997, Nd 6105 -Mk 12657, IUCN: nt.

*Astragalus sachanewii Sirj.: rocky slopes, locality 53; 16.07.1997, Nd 6017 -Mk12580, IUCN: nt.

*Astragalus bashkalensis Chamberlain: meadows, locality 29; 19.07.1997, Nd 6095 -Mk 12657, rocky slopes locality 34; 22.06.1997, Mk 12126-Nd 5554; steppe, locality 37; 27.06.1997, Mk 12073-Nd5502, Mk 12289Nd 5717; steppe, locality 38 ; 27.06.1997, Nd 5693, 5704 -Mk 12265,12276; rocky slopes, locality 54; 27.07.1997, Nd 6280 -Mk 12843, IUCN: vu. Variations: Legume; c. $16 \mathrm{~mm}$ villous with long white and short black hairy, ovoid sessile to short stipitate ( legume unknown).

*Astragalus tauricolus Boiss.: steppe, locality 7; 22.05.1997, Mk 11681, IUCN: lc.

*Astragalus pinetorum Boiss.: steppe slopes, locality 27; 21.06.1997, Nd 5121 -Mk 11629, IUCN: lc.

*Astragalus icmadophilus Hand.-Mazz.: rocky slopes, locality 53; 16.07.1997, Nd 5996, IUCN: 1c.

*Astragalus rechingeri Sirj.: road slopes, locality 50; 15.07.1997, Nd 5910 -Mk 12474; rocky slopes, locality 53 ; 16.07.1997, Nd 5996 -Mk 12559. IUCN: nt. Variations: Calyx black and white hairy (sparsely black hairy).

*Astragalus trifoliastrum Hub.-Mor. \& Matthews: steppe slopes, locality 23; 18.06.1997, Mk 11933-Nd 5362; rocky slopes, locality 26; 19.06.1997, Mk 11994-Nd 5422; locality 36; 26.06.1997, Mk 12210- Nd 5638, IUCN: nt. 
*Astragalus ermineus Matthews: rocky slopes, locality 53; 16.07.1997, Nd 5902a, 6032 -Mk 12466a, 12595, IUCN: cd.

*Astragalus halicacabus Lam.: meadows, locality 43; 12.07. 1997 Nd 5785 -Mk 12534, IUCN: lc.

Astragalus gymnalopecias Reich. fil.: locality 42; 15.06.1999, Mf 1599, IUCN: en.

*Astragalus asciocalyx Bunge : locality 25; 18.06.1997, Mk 11931-Nd 5360; rocky slopes, locality 26; 19.06.1997, Mk 11978-Nd 5407,IUCN: lc.

Astragalus karamasicus Boiss. \& Balansa: steppe, locality 105; 08.06.1991 B 1731, IUCN: lc.

Astragalus lycius Boiss.: steppe, locality 106; 28.05.2002, Ok 2997, IUCN: lc.

*Astragalus fumosus Boriss. : around road, locality 28; 21.06.1997, Mk 12030-Nd 5458, IUCN: nt.

Astragalus xerophilus Ledeb.: steppe, locality 107; 12.07.2007, Ok 6773, IUCN: nt.

Astragalus bicolor Lam.: steppe, locality 108; 26.06.2005, Ok 3941.

*Astragalus globosus Vahl: locality 11; 25.05.1997, Nd 6426k, IUCN: lc.

Astragalus armeniacus Boiss.: steppe, locality 109; 07.06.2003, Mü 8282.

Astragalus schizopterus Boiss.: steppe, locality 109; 07.06.2003, Mü 8233.

Astragalus nitens Boiss. \& Heldr.: steppe, locality 177; 07.06.2003, Mü 8217.

*Astragalus campylosema Boiss. subsp. nigripilis Hub.Mor. \& Chamb.: steppe, locality 3; 10.08.1996, Nd 5061a, IUCN: lc.

*Astragalus cinereus Willd.: steppe, locality 21; 13.06.1997,Mk 11923-Nd 5353; meadows, locality 29; 19.07.1997, Nd 6093 -Mk 12655; rocky slopes, locality 34; 22.06.1997, Mk 12107b, 12135-Nd 5538 b, 5563; steppe, locality 38; 27.06.1997, Mk 12222-Nd 5650; meadows, locality 47; 13.07.1997, Nd 6143 -Mk 12706; rocky crevices, locality $58 ; 28.05 .2007, \mathrm{Mk} 12065-\mathrm{Nd}$ 5492, IUCN: 1c.

*Astragalus davisii Chamb. \& Matthews: locality 1; 04.06.1996, Nd 4991; rocky slopes, locality 18; 08.06.1997, Mk 11821-Nd 5252; road slopes, locality 50; 15.07.1997, Nd 5930 -Mk 12494, IUCN: cd. Variations:Legume c. 20 × $9 \mathrm{~mm}$ white tomentose to felted hairy (unknown)

*Astragalus chaldiranicus Kit Tan \& Sorger: meadows, locality 46; 13.07.1997, Nd 5839, 5860 -Mk 12400, IUCN: vu.

*Astragalus comosoides Chamb. \& Matthews: meadows, locality 8; 24.05.1997, Nd 5155, 7065, IUCN: lc.

Astragalus bahcesarayensis Akan, Frrat \& Ekici: stony places, locality 62; 05.08.2004, Mf 4221.

Cicer pinnatifidum Jaub. \&Spach: rocky slopes, locality 110; 04.05.2003, Aa 2956.
Vicia alpestris Stev. subsp. hypoleuca (Boiss) P.H. Davis: steppe, locality 111; 12.07.2007, Ok 6852., IUCN: lc.

*Lathyrus brachypterus Cel. var. haussknechtii (Sirj) D P.H. Davis: meadows, locality 19; 12.06. 1997, Mk 11855- Nd 5285, IUCN: lc.

Lathyrus nivalis Hand.-Mazz.: rocky slopes, locality 82; 21.06.2000, Mf 2590, IUCN: lc.

Trifolium longidentatum Nábělek: meadow, rocky, locality $201 ; 18.08 .2002$, M 7952b,

Hedysarum vanense Hedge \& Hub.-Mor.: steppe, locality 204; 10.06.2007, İd 443, IUCN: vu.

*Hedysarum cappadocicum Boiss.: steppe, locality 38; 27.06.1997, Mk 12227-Nd 5655, IUCN: lc.

*Hedysarum erythroleucum Boiss.: rocky slopes, locality 53; 16.07.1997, Nd 6026- Mk12562, IUCN: 1c.

Onobrychis stenostachya Freyn subsp. sosnowskyi (Grossh.) Hedge: steppe, locality 205; 26.07.1997, Fö 5386, IUCN: vu.

Onobrychis fallax Freyn \& Sint.: steppe meadows, locality 4; 22.07.2001, Ma.1217, IUCN: 1c.

Onobrychis huetiana Boiss.: meadow, locality 208; 31.05.2008, Da 1222, IUCN: nt.

\section{FAM: ROSACEAE}

Prunus kurdica Fenzl ex Fritsch: roadside, locality 206; 14.07.2001, Tç 747, IUCN: en.

*Potentilla anatolica Peşmen: meadows, locality 29; 19.07.1997, Nd 6057- Mk 12620; meadows, locality 43; 12.07. 1997, Nd 5781- Mk 12342; rocky slopes rocky slopes, locality 54; 27.07.1997, 27.07.1997, Nd 6231 -Mk 12795, IUCN: lc.

Potentilla armeniaca Siegfr. ex Th.Wolf steppe, locality 209; 05.08.2007, Ok 7533, IUCN: vu.

*Rosa pisiformis (Christ.) D. Sosn.: around road, locality 55; 19.07.1997, Nd 7056, IUCN: nt.

\section{FAM: LYTHRACEAE}

Lythrum anatolicum Leblebici \& Seçmen subsp. vanense Pınar: wet and sandy places, locality 63; 25.8.2002, Mp 1229

\section{FAM: CRASSULACEAE}

Rosularia davisii Muirhead: rocky place, locality 112; 03.07.2003, Mü 8404a, IUCN: cd.

Sempervivum armenum Boiss\&Huet var. armenum: steppe rocky place, locality $114 ; 15.07 .2002$, Ok 3561 , IUCN: lc.

\section{FAM: GROSSULARIACEAE}

Ribes anatolica Behçet: moving stone area, locality 64; 01.08.2013, B 318.

\section{FAM: APIACEAE}

Chaerophyllum hakkiaricum Hedge\&Lamand.: steppe, locality 115; 18.07.2001, Mü 6110, IUCN: vu.

*Prangos uechtritzii Boiss. \& Hausskn.: meadows, locality 29; 19.07.1997, Nd 6102 -Mk12664, IUCN: lc. 
Cymbocarpum wiedemanii Boiss.: arable field place, locality 116; 21.06.2008, İd 1369, IUCN: cd.

Diplotaenia turcica Pimenov \& Kljuykov: moving stone area, locality 65; 01.08.2013, Mf 30469, IUCN: nt.

Laserpitium carduchorum Hedge \& Lemond.: arable field place, locality 116; 21.06.2008, Mp 2537.

Malabaila lasiocarpa Boiss.: steppe, locality 150; 29.06.2006,Ok4791, IUCN: lc.

Heracleum crenatifolium Boiss.: the slopes of the valley, locality 118; 29.06.2006, Ok 4884, IUCN: nt.

Trigonosciadium tuberosum Boiss.: steppe, locality 119; 21.06.2007, Ok 6713, IUCN: dd.

Trigonosciadium intermedium Freyn\&Sint.: oak clearance slope, locality 120; 09.06.2012, He 1020, IUCN: en.

\section{FAM: VALERIANACEAE}

Valerianella glomerata Boiss \& Bal.: locality 121; 28.06.1997, Fö 4280, IUCN: lc.

\section{FAM: DIPSACACEAE}

Cephalaria anatolica Shkhiyan: steppe, locality 151; 18.07.2008, Da 1314, IUCN: cr

Cephalaria sparsipilosa Matthews: meadows, locality 122; 30.07.2007, Ok 7384, IUCN: cd.

Scabiosa rufescens Freyn \& Sint.: meadows, locality 152; 25.06.2002, Mü 7274, IUCN: cd.

\section{FAM: ASTERACEAE}

Inula helenium L. subsp. orgyalis (Boiss.) Grierson: meadow, locality $123 ; 25.08 .2007$, Ok7627, IUCN: nt.

Pulicaria armena Boiss. \& Kotschy: the edge of Zilan Creek, locality 153; 07.08.2006, Ok5179, IUCN: lc.

Helichrysum compactum Boiss.: steppe, locality 172; 07.08.1996, Fö 2575, IUCN: en.

Helichrysum arenarium (L.) Moench subsp. aucheri (Boiss.) P.H. Davis \& Kupicha: steppe, locality 173; 07.06.2008,İ 1185, IUCN: lc.

Helichrysum arenarium (L.) Moench subsp. erzincanicum P.H. Davis \& Kupicha: steppe, locality $174 ;$ 16.08.2007 Ok 7580, IUCN: vu.

*Anthemis wiedemanniana Fish. \& Mey.: meadows, locality 5; 03.06.1997, Nd 7076, IUCN: lc.

Anacyclus anatolicus Behçet \& Almanar: steppe, locality 175; 09.05.2004, Тç 92,

Achillea cappadocica Hausskn. \& Bornm.:alpine steppe, locality 178; 20.07.1988, B1317, IUCN: lc.

*Tanacetum zahlbruckneri (Nab.) Grierson: rocky slopes, locality 34; 22.06.1997, Mk 12121,12166Nd 5549,5494; road slopes, locality 50; 15.07.1997, Nd 5926 -Mk 12490, IUCN: 1c.

Tanacetum heterotomum (Bornm.) Grierson: steppe, locality 182; 25.05.1996, Fö 393, IUCN: vu.

Tanacetum nitens (Boiss. \& Noë) Grierson: steppe-rocky areas, locality 183; 08.07.2001, Ok 2144, IUCN: lc.
Tanacetum cadmeum(Boiss.) Heywood subsp. orientale Grierson: steppe fields, locality 1194; 23.07.2001, Mü 5968, IUCN: 1c.

*Tripleurospermum callosum (Boiss. \& Heldr.) E. Hossain: road slopes, locality 50; 15.07.1997, Nd 592512489, IUCN: lc.

*Tripleurospermum monticolum (Boiss. \& Huet) Bornm.: meadows, locality 46; 13.07.1997, Nd 5831 -Mk 12392, IUCN: 1c.

*Cousinia bicolor Freyn \& Sint.: rocky slopes, locality 26; 19.06.1997, Mk 12016, Nd 5444, IUCN: lc.

*Cousinia eriocephala Boiss. \& Hausskn.: road slopes, locality 50; 15.07.1997, Mk 100798, IUCN: lc.

Cousinia nabelekii Bornm.: steppe, locality 6; 22.07.2001, Ma 1605, IUCN: nt.

Cousinia hakkarica Hub.-Mor.: steppe, locality 179; 31.07.2002, Mü 7809, IUCN: vu.

*Cousinia vanensis Hub.-Mor.: meadows, locality 8; 24.05.1997, Nd 7009, IUCN: lc.

Cirsium peshmenianum Y1ld1z, Dirmenci \& Arabac1: conglomerate locality 68; 18.08. 2008, BY 16958

*Carduus lanuginosus Willd.: rocky slopes meadows, locality 47; 13.07.1997, Nd 6215, IUCN: lc. Variations: Capitula to $4 \times 4 \mathrm{~cm}(2-3 \times 2-3 \mathrm{~cm})$, spines $1-7 \mathrm{~mm}(2-10$ $\mathrm{mm})$.

Gundelia dersim Vitek, Yüce \& Ergin: steppe areas, locality 66; 10.5.2017, Mf 33729

Gundelia colemerikensis Firat: dry steppe fields, locality 67; 10.05.2017, Mf 33744

Jurinea cataonica Boiss.\& Hausskn.: steppe, locality 180; 12.07.2007, Ok6790, IUCN: lc.

*Centaurea saligna (C.Koch) Wagenitz: creek edge steppe, locality 51; 16.07.1997, Mk 12884, IUCN: lc.

Centaurea sessilis J.F.Gmel.;steppe, May 1986, Hö 1745, IUCN: lc.

Centaurea armena Boiss.: steppe, locality 185; 12.07.2007, Ok6912, IUCN: lc.

Centaurea demirizii Wagenitz: steppe, locality 186; 30.06.1998, Mü3468, IUCN: vu.

Centaurea fenzlii Reichardt: steppe, locality 187; 29.07.2007, Ok7289, IUCN: 1c.

Centaurea lydia Boiss.: locality 193; 09.06.1996 Fö964, IUCN: cd.

Echinops pungens Trautvt. var. adenoclados Hedge: steppe, locality 195; 01.08.2001, M6411 195, IUCN: nt.

Echinops orientalis Trautv.: steppe, locality 202; 19.10.1997, M2240,.

Scorzonera semicana DC.: meadows, locality 203; 26.05.2007 Ok5940, IUCN: lc

*Scorzonera eriophora DC.: creek edge steppe, locality 51; 16.07.1997, Mk 12881, IUCN: lc.

Tragopogon aureus Boiss.: steppe, locality 207; 11.06.2001, Ok1639, IUCN: lc. 
Tragopogon vanensis Gültepe, Coșkunç. \& Makbul: locality 69; 08.04.2010, $\mathrm{Mg} 267$.

Taraxacum davisii Van Soest.: meadow area, locality 210; 5.05.2002, Ok2890, IUCN: en.

*Crepis dioritica Schott. \& Ky. ex Boiss.: rocky slopes, locality 53; 16.07.1997, Nd 5949 -Mk 12514, IUCN: lc.

*Crepis macropus Boiss. \& Heldr.: meadows, locality 29; $\mathrm{Nd} 6110$-Mk 12672; rocky slopes, locality 54; 27.07.1997, Nd 6222- Mk IUCN: lc.

*Crepis armena DC. : rocky slopes, locality 34; 22.06.1997, Mk 12110-Nd 5539, rocky slopes, locality 53; 16.07.1997, Nd 6034- Mk 12597, IUCN: lc.

Crepis gemicii Yıldırım, Bingöl \& Armağan: meadows and openings in Quercus petraea subsp. pinnatiloba, a.s.l. locality $70 ; 17.07 .2002$, Öb 12199.

Psephellus vanensis A. Duran, Behçet \& B. Doğan: steppe fields, a.s.1, locality 71; 17.06.2009, B\&A 1603

\section{FAM: CAMPANULACEAE}

*Campanula coriacea P.H. Davis: basalt rocky slopes, locality 45; 13.07.1997, Mk 12415-Nd 5852, IUCN: lc.

Campanula hedgei P.H.Davis: steppe, locality 154; 09.08.1998, Fö 5480, IUCN: cd.

*Campanula bornmuelleri Nab.: steppe, locality 25; 18.06.1997, Mk11955-Nd5385; rocky slopes, locality 48; 15.07.1997, Mk11768; rocky slopes, locality 53; 16.07.1997, Nd5948, 6016 -Mk 12512, 12579; rocky slopes, locality 54; 27.07.1997, Nd 6240- Mk 12804, IUCN: cd. Variations: Some samples; Leaves glabrous both sides (leaves hirsute both sides) and stem usually 2-4 flowered (usually one flowered).

*Campanula saxonorum Gandoger: steppe slopes, locality 14; 05.06.1997, Mk 11772, IUCN: lc. Variations: Stems, 5-20 cm [(10) $15-25(30) \mathrm{cm}]$.

Asyneuma limonifolium (L.) Janch. subsp. pestalozzae (Boiss.) Damboldt: steppe, locality 124; 07.07.1997, Fö 5172, IUCN: 1c.

Asyneuma trichostegium (Boiss.) Bornm.: meadows, locality 155; 14.08.1997, Fö 1617, IUCN: dd.

Asyneuma linifolium (Boiss. \& Heldr.) Bornm. subsp. eximium (Rech.f.) Damboldt: steppe, locality 156; 17.07.1997, Mü 1672.

\section{FAM: CONVOLVULACEAE}

*Convolvulus galaticus Rostan ex Choisy: valley slopes, locality 49; 15.07.1997,Nd 5861- Mk12424, IUCN: lc.

\section{FAM: BORAGINACEAE}

Cynoglossum vanense Sutorý : locality 72; 22.07. 1998 Mü 4533

Rochelia disperma (L.f.) K.Koch var. microcalycina (Bornm.) Edmondson: steppe, locality 168; 12.07.2007, Ok 6822, IUCN: lc.

Myosotis platyphylla Boiss: meadows, locality 181; 25.08.2007, Ok 7662, IUCN: vu.

*Rindera caespitosa (A.DC.) Bunge: plateau, locality 22; 02.04.1999, Mf 1045, IUCN: lc.
Onosma neglecta Riedl: meadow area, locality 188; 20.07.2005, Tç 825, IUCN: cd.

*Onosma polioxantha Rech. f.: rocky slopes, locality 26; 19.06.1997, Nd 5438, 6337a, IUCN: lc.

Onosma argentata Hub- Mor.: steppe, locality 189; 28.06.1996, Fö 2542, IUCN: vu.

Onosma velutina Boiss.: steppe, locality 190; 10.06.1987, B 710, IUCN: cd.

Onosma procera Boiss: steppe, locality 191; 26.06.1997. Fö 3633, IUCN: nt.

*Onosma isauricum Boiss. \& Heldr.: Çatak, valley slopes, locality 49; 15.07.1997,Nd 5864 -Mk 12427, IUCN: lc.

Onosma bracteosa Hausskn. \& Bornm.: wet meadows, locality 13; 10.06.2001, Ma1309.IUCN: lc.

*Onosma mutabile Boiss.: meadows, locality 47; 13.07.1997, Mk 11924- Nd 5354, IUCN: lc. Variations: Stems up to 8 (1-4) some basal leaves not widest (basal leaves widest).

Onosma armenaDC.: steppe, locality 192: 19.07.1986, Hö 878, IUCN: lc.

Onosma proballanthera Rech. fil.: rocky slopes, locality 20; 23.09.200, Ma. 2129, IUCN: cd.

Anchusa leptophylla Roemer \& Schultz subsp. incana (Ledeb.) D. F. Chamb.: creek edge, locality 196; 17.06.2006, Ok 5440, IUCN: cd.

Nonea karsensis M.Popov:: wheat field, locality 197; 02.08.1996, F 616, IUCN: dd.

Nonea stenosolen Boiss. \& Balansa: steppe, locality 198; 10.05.1998, M 2362, IUCN: lc.

Alkanna tubulosa Boiss.: alpine meadow, locality 199; 10.05.1998, B 687, IUCN: lc.

Alkanna froedinii Rech.fil.: border plateau, locality 200; 18.07.1997, Fö5870, IUCN: 1c.

\section{FAM: SOLANACEAE}

*Lycium anatolicum A. Baytop \& R. Mill: steppe slopes, locality 23; 18.06.1997, Mk, 11962-Nd 5392, IUCN: lc.

\section{FAM: SCROPHULrIACEAE}

Verbascum kurdicum Hub.-Mor.: steppe, locality 125; 25.05.2002, Aa 2342, IUCN: 1c.

Verbascum oreophilum C. Koch var. oreophilum: meadow, locality 157; 12.06.2005, Tç 589, IUCN: lc.

Verbascum vanense Hub.-Mor.: steppe, locality 126; 21.06.2001, Ok1787, IUCN: cd.

Verbascum golawanense Firat: fallow fields, edge of fields, locality 73; 01.07.2011, Mf 27749.

Rhynchocorys kurdica Nábělek: plateau, moist area, locality $127 ; 26.06 .2005$, B718.

*Scrophularia pulverulenta Boiss. \& Nöe: Çatak, slopes, locality 49; 15.07.1997, Nd 5876 -Mk 12439, IUCN: lc.

*Scrophularia libanotica Boiss. subsp. libanotica var. urartuensis R. Mill: rocky slopes, locality 2; 03.08.1996 
Nd 5001; rocky slopes, locality 18; 08.06.1997, Mk 11838-Nd 5268; steppe, locality 21; 13.06.1997, Mk 11925-Nd 5355; wet meadows, locality 24; 23.07.1997, Mk 11960-Nd 5390; rocky slopes, locality 26; 19.06.1997, Mk 1190-Nd 5418; valley slopes, locality 49; 15.07.1997, Nd 5876 -Mk12439, Nd 5962,5994- Mk 12525, 12557, IUCN: lc.

Scrophularia versicolor Boiss.: steppe, locality 128; 10.08.1996, Fö 2630, IUCN: vu.

Chaenorhinum minus (L.) Lange subsp. anatolicum P.H.Davis: sandy area, locality 129; 26.07.2007, İd 819, IUCN: lc.

Linaria genistifolia (L.) Miller subsp. confertiflora (Boiss.) P.H.Davis: steppe, locality 158; 29.06.1996, Fö 2684, IUCN: lc.

Linaria corifolia Desf.: steppe, locality 159; 05.10.1997, Fö 1440 , IUCN: lc.

Veronica fridericae M.A. Fischer: meadows, locality 32; 21.07.2002, Ma 2874,IUCN: cd.

Bungea trifida (Vahl) C.A.Meyer: steppe, locality 130; 07.06.2002, Ma 2452.

\section{FAM: LAMIACEAE}

Ajuga bombycina Boiss.: steppe, locality 131; 15.05.1996, Fö 1164, IUCN: nt.

*Scutellaria orientalis L. subsp. santaloides (Hausskn. ex Bornm.) Edmonson: locality 33; 03.07.1997, Nd 5729, IUCN: 1c.

*Phlomis armeniaca Willd.: Özalp road 20. Km, slopes, locality 36; Mk 12201- Nd 5629; meadows, locality 47; 13.07.1997, Nd 6135- Mk12967, IUCN: lc.

*Marrubium parviflorum Fisch. \& C. A. Mey. subsp. oligodon (Boiss.) Seybold: locality 55; Nd 6050- Mk 12613; steppe, locality 57; 19.07.1997, Nd 6211- Mk 12775, IUCN: lc.

Marrubium vanense Hub.-Mor.: steppe, locality 132; 29.07.2006, Mf627, IUCN: en.

Marrubium vulcanicum Hub.-Mor.: steppe, locality 133; 23.07.2003, Mü 8826, IUCN: vu.

Stachys ramosissima Montbret \& Aucher ex Benth.var. ramosissima: steppe, locality 160; 29.06.1997, Fö 4444 , IUCN: cd.

Lophanthus turcicus Dirmenci, Y1ld1z \& Hedge: rocky and stony north slope, locality 74; 24.07.2009, Td 3707

*Micromeria cristata (Hampe) Griseb. subsp. orientalis P.H. Davis: creek edge steppe, locality 51; 16.07.1997, Nd 5933 -Mk 12497, IUCN: lc.

Micromeria cremnophila Boiss. \& Heldr. subsp. anatolica P.H.Davis: rocky cracks, locality 167; 15.07.2003, Mü8595, IUCN: lc.

*Cyclotrchium glabrescens (Boiss. \& Kotschy ex Rech. fil.) Leblebici: creek edge steppe, locality 51; 16.07.1997, Mk 12883, Nd 5936 -Mk 12500, IUCN: cd.

*Thymus brachychilus Jalas: rocky slopes, locality 53; 16.07.1997, Nd 5979 -Mk 12542, IUCN: lc.
* Salvia kronenburgii Rech. fil.: steppe slopes, locality 27; 21.06.1997, Mk11201-Nd 5449, IUCN: vu.

Salvia longipedicellata Hedge.: steppe, locality 139; 22.05.2002, Mü 7015, IUCN: nt.

Salvia dichroantha Stapf.: steppe, locality 140; 09.06.1997, Fö1899, IUCN: lc.

\section{FAM: PLUMBAGINACEAE}

*Limonium vanense Kit-Tan \& Sorger : meadows, locality 46; 13.07.1997, Nd 5848 -Mk 12411, IUCN: vu.

Limoniopsis davisii Bokhari: rocky and stone steppe, locality 161; 03.07.2003, Mü8413, IUCN: en.

Acantholimon venustum Boiss. var. assyriacum ( Boiss.) Boiss.: steppe, locality 162; 16.07.2006, Ok5007, IUCN: nt.

Acantholimon acerosum (Willd.) Boiss. var. brachystachyum Boiss.: steppe, locality 170; 14.07.2001, Mü5224a, IUCN: vu.

Acantholimon calvertii Boiss.: rocky steppe, locality 171; 08.07.2001, Ok2075, IUCN: 1c.

*Acantholimon reflexifolium Bokhari :rocky slopes, locality 54; 27.07.1997, Nd 6235 -Mk 12799, IUCN: nt.

Acantholimon bashkaleicum Doğan \& Akaydın: steppe, locality 75; 06.07.2002, D\&A 7544.

Acantholimon artosense Doğan \& Akaydın: steppe, locality 76; 05.07.2002, D\&A 7532.

Acantholimon hoshapicum Doğan \& Akaydın:steppe, locality 77; D\&A 7540.

\section{FAM: PLANTAGINACEAE}

*Plantago anatolica Tutel \& R. Mill.: meadows, locality 19; 12.06.1997, Mk 11850- Nd 5280, IUCN: cd.

\section{FAM: EUPHORBIACEAE}

*Euphorbia grisophylla M. L. S. Khan: plateau, locality 22; 02.04.1999, Mf 2062, IUCN: lc.

Euphorbia falcata L. subsp. macrostegia (Bornm.) O.Schwartz: steppe, locality 113; 08.09.2007, İ 848, IUCN: lc.

\section{FAM: FAGACEAE}

*Quercus petraea (Mattuschka) Liebl. subsp. pinnatiloba (C. Koch) Menitsky: steppe, locality 42;15.06.1999,Mf 1776, 53;Mf1542, IUCN: lc.

\section{FAM: RUBIACEAE}

Galium margaceum Ehrend. \& Schönb.-Tem. : rocky slope, locality 134; 20.06.1992, Fö 1404, IUCN: lc.

\section{FAM: LILIACEAE}

*Allium microspatum Ekberg: rocky slopes, locality 54; 27.07.1997, Mk:12785-Nd 6221, IUCN: cd.

Allium tauricola Boiss.: rocky slopes, locality 166; 04.08.2001, Mü 6533, IUCN: lc.

*Allium stearnianum Koyuncu, Özhatay \&Kollmann subsp. vanense Kollmann \&Koyuncu : wet meadows, locality 24; 23.07.1997,Mk 12861-Nd 6298; steppe, 
locality $38 ; 27.06 .1997$, Mk 12280-Nd 5708; : meadows, locality 47; 13.07.1997, Mk 12685-Nd 6123; road slopes, steppe, locality 52; 16.07.1997, Mk 12508-Nd 5944; rocky slopes, locality 53; 16.07.1997, Mk 12545-Nd 5982; steppe, locality 57; 19.07.1997, Mk 12772-Nd 6208; steppe, locality 41; 19.07.1997, Mk 12769-Nd6205, IUCN: nt.

*Allium shatakiense Rech. fil.: wet meadows, locality 35; 22.06.1997, Mk 12132-Nd 5560, IUCN: nt.

Allium armenum Boiss. \& Kotschy: rocky steppe, locality 141; 02.09.2007, Ok 7833, IUCN: lc.

Allium hoshabicum Frrat: Holotype, steppe and meadow, elevation, locality 78; 21.07.2012, Mf 28979.

Puschkinia bilgineri Yıldırım: alpine meadows, locality 79; 19.06.2014, Hy 2695.

*Bellevalia rixi Wendelbo: meadows, locality 9; 24.05.1997 Mk 11704-Nd5170; steppe, locality 21; 13.06.1997, Mk 11894-Nd5324, locality 37; Mk 12069Nd 5498: steppe, locality 38; 27.06.1997, Mk 12279-Nd 5707, IUCN: en.

*Fritillaria michailovskyi Fomin: steppe slopes, locality 16; 07.06.1997, Mk 11798-Nd 5229, IUCN: cd.

*Fritillaria minima Rix: rocky slopes, locality 12; 26.05.1997, Mk 11736-Nd 5200, steppe slopes, locality 16; 07.06.1997, Mk 11798-Nd 5229, rocky slopes, locality 53; 16.07.1997, Mk 12520- Nd 5957, IUCN: vu.

Tulipa koyuncui Eker \& Babaç: steppe, locality 80; 13.05.2010, E 1565 .

Gagea vanensis Tekşen \& Karaman: rocky slopes, locality $81 ; 21.06 .2000$, Mt 2460

\section{FAM: IRIDACEAE}

*Iris sari Schott ex Baker: road slopes, locality 40; 16.05.1999, Mf 1292, IUCN: lc.

\section{FAM: ORCIDACEAE}

Dactylorhiza osmanica (Klinge) P.F.Hunt \& Summerh. subsp. osmaniaca: the edge of Zilan creek, locality 163; 29.05.2006, Ok 4386. IUCN: lc.

\section{FAM: CYPERACEAE}

Carex distans L.: meadows, locality 136; 07.06.2003, Mü 8309 a

\section{FAM: POACEAE}

Bromus macrocladus Boiss.: steppe, locality 137; 17.06.2007, Ok 631, IUCN: en.

Festuca anatolica Markgr.-Dann. subsp. borealis Markgr.-Dannenb.: steppe, locality 164; 08.06.1991, B1696, IUCN: cd.

Festuca anatolica Markgr.-Dann. subsp. anatolica: steppe, locality 165; 30.06.1998, Mü 3749, IUCN: lc.

Eremopoa mardinensis R.Mill: steppe, locality 138; 30.06.1990, B 3204, IUCN: en.

\section{Discussions}

With this study, 259 endemic taxa belonging to 34 families and 117 genera were compiled in the Lake Van Basin. Twenty three of them, within 20 genera, are unique to the Lake Van Basin.

The distribution of 117 genera and 259 taxa within 34 families are as follows: Asteraceae- 20 genera 41 taxa; Fabaceae 7 genera 40 taxa; Brassicaceae- 9 genera 29 taxa; Boraginaceae - 8 genera 20 taxa; Caryophyllaceae5 genera, 20 taxa; Lamiaceae- 10 genera 15 taxa; Scrophulriaceae- 7 genera 13 taxa; Liliaceae- 6 genara 12 taxa; Apiaceae- 8 genera 9 taxa; Plumbaginaceae- 3 genera, 9 taxa; Ranunculaceae; 2 genera 9 taxa; Campanulaceae- 2 genera 7 taxa; Poaceae- 3 genera 4 taxa; Rosaceae- 3 genera 4 taxa; Dipsacaceae- 2 genera 3 taxa; Geraniaceae- 2 genera 3 taxa; Crassulaceae- 2 genera 2 taxa; Papaveraceae- 2 genera 2 taxa; Euphorbiaceae-1genus 2 taxa; Linaceae-1genus 2 taxa; Convolvulaceae- 1 genus 1 taxon; Cyperaceae- 1 genus 1 taxon; Fagaceae- 1 genus 1 taxon; Grossulariaceae- 1 genus 1 taxon; Hypericaceae- 1 genus 1 taxon; Iridaceae-1 genus 1 taxon; Lythraceae-1 genus 1 taxon; Orcidaceae- 1 genus 1 taxon; Plantaginaceae- 1 genus 1 taxon; Polygonaceae- 1 genus 1 taxon; Rubiaceae- 1 genus 1 taxon; Rutaceae- 1 genus 1 taxon; Solanaceae- 1 genus 1 taxon; Valerianaceae- 1 genus 1 taxon.

Most of the endemic taxa have been located around Güzeldere pass (Çuh pass), Artos, Kavuşşahap and Ispiriz mountains respectively (Davis et al., 1988; Ekim, 2005; Özhatay et al., 2009).

Among the endemic taxa, Acantholimon artosense, Acantholimon bashkaleicum, Acantholimon hoshapicum, Astragalus bahcesarayensis, Cirsium peshmenianum, Crepis gemicii, Dianthus vanensis, Draba orientalis, Fritillaria minima, Gagea vanensis, Limoniopsis davisii, Limonium vanense, Lophanthus turcicus, Lythrum anatolicum subsp. vanense, Psephellus vanensis, Puschkinia bilgineri, Ranunculus vanensis, Ribes anatolica, Silene acaulis subsp. vanensis, Silene miksensis, Tragopogon vanensis, Tulipa koyuncui and Verbascum golawanense are unique to Lake Van basin.

IUCN Red Data Categories (if known) of 259 taxa in our study are as follows (Ekim et al. 2000): IUCN: Lr (lc) 118, IUCN: Lr (cd) 33, IUCN: Lr (nt) 28, IUCN: vu 20, IUCN: en 16, IUCN: dd 4, IUCN: cr 1. IUCN Red Data categories of 40 taxa are unknown.

\section{Acknowledgments}

We would like to thank to The Scientific and Technical research Council of Turkey, Van Yüzüncü Y1l University Research Council and The Environment Management of Van Province for their financial supports. We are also grateful to Prof. Dr. Abdullah KAYA for his help in arranging the article, and Prof. Dr. Tuna EKIMM for their help.

\section{References}

Akan H, Firat M, Ekici M (2008). Astragalus bahcesarayensis (Leguminosae-Papilionoideae), a new species of section Alopecuroidei DC. from Turkey. Botanical Journal of the Linnean Society156: 439-444. 
Atalay İ (1999). Türkiye Coğrafyası. İzmir: Ege Üniversitesi Basım Evi.

Behçet L (2001). A New Species of Ribes L. (Grossulariaceae) from East Anatolia,Turkey. Turkish Journal of Botany 25: 103105.

Bulut Z, Y1lmaz H (2010). The current situation of threatened endemic flora in Turkey: Kemaliye (Erzincan) Case. Pakistan Journal of Botany 42(2): 711-719.

Çiftçi Y, Işık MA, Alkevli T, Yeşilova Ç (2008). Van Gölü Havzasının çevre jeolojisi. Jeoloji Mühendisliği Dergisi 32(2): 45-77.

Davis PH (1965-1982). Flora of Turkey and East Aegean Islands, Vol: I-IX, Edinburgh: Edinburgh University Press.

Davis PH, Tan K, Mill RR (1988). Flora of Turkey and the East Aegean Islands (suppl. 1). Vol. 11. Edinburgh: Edinburgh University Press.

Degens ET, Wong HK, Kempe S, Kurtman F (1984). A geological study of lake van, Eastern Turkey. Geologische Rundschau 73(2): 701-734

Dirmenci T, Yıldız B., Hedge IC, Firat M (2010). Lophanthus (Lamiaceae) in Turkey: a new generic record and a new species, Turk J Bot 34:123-129.

Doğan B, Behçet L, Duran A, Avlamaz D (2015). Psephellus vanensis (Asteraceae),a new species from east Turkey. PhytoKeys 48: 11-19.

Doğan M, Akaydın G (2004). Three new species with two flowered spikelets in Acantholimon (Plumbaginaceae) from East Anatolia, Turkey. Botanical Journal of the Linnean Society 144: 497-505.

Eker İ, Babaç TM (2010). Tulipa koyuncui Eker \& Babac, sp. nov. (Liliaceae) from east Anatolia, Turkey. Nordic Journal of Botany 28: 324-328.

Ekim T (2005). Bitkiler, Tohumlu Bitkiler, Türkiye’nin Biyolojik Zenginlikleri. Ankara: Türkiye Çevre Vakfi.

Ekim, T, M. Koyuncu, M. Vural, H. Duman, Z. Aytaç and N. Adıgüzel. 2000. Red Data Book of Turkish Plants (Pteridophyta and Spermatophyta). Turkish Association for the Conservation of Nature \& Van Centennial University, ISBN: 97593611-0-8, Ankara: Barışcan Ofset.

Erik S ve Tarıkahya B. (2004). Türkiye florası üzerine. Kebikeç İnsan Kaynakları Araştırmaları Dergisi 17:139-163.

Firat M (2017a). Allium hoshabicum a new species of A. sect. Codonoprasum (Amaryllidaceae) from Van (Turkey). Phytotaxa 312(1): 129-134.

Firat M (2017b). Verbascum golawanense (Scrophulariaceae), a new species from Van, Turkey. Phytotaxa 305(1): 21-28.

Firat M, Yıldız K (2016). Silene miksensis (Caryophyllaceae), a new species from eastern Anatolia. Phytotaxa 273(4): $283-292$.

Gültepe M, Çoşkunçelebi K, Makbul S, Terzioğlu S (2016). Taxonomic notes on Tragopogon, and two newly described taxa from Anatolia. Nordic Journal of Botany 34: 529-537.

İlçim A, Behçet L, Mükemre M (2013). Dianthus vanensis (Caryophyllaceae), a new species from Turkey. Turkish Journal of Botany 37: 219-224.

Mgm, İllere Ait Mevsim Normalleri (2018). https://www.mgm.gov.tr/ [Accessed 15 May 2018].

Karabacak O, Behçet L (2009). Draba orientalis (Brassicaceae), a new species from Turkey. Annales Botatici Fennici 46: 447450.

Med Plant Base (2018). http://ww2.bgbm.org/EuroPlusMed/query.asp/ [Accessed 16 May 2018].

Özgökçe F, Tan K, Stevanović V (2005). A new subspecies of Silene acaulis (Caryophyllaceae) from East Anatolia, Turkey. Annales Botatici Fennici 42: 143-149.

Özhatay N, Kültür, S ve Aslan, S. (2009). Check-list of additional taxa to the supplement flora of Turkey IV. Turkish Journal of Botany 33:191-226.

Pınar SM (2017). Lythrum anatolicum subsp. vanense (Lythraceae): a new subspecies from Eastern Anatolia, Turkey. Acta Biologica Turcica 30(1): 16-19.

Sutorý K (2005). A new Species of Cynoglossum (Boraginaceae-Cynoglosseae) from Eastern Turkey. Edinburgh Journal of Botany 61(2\&3): 119-126.

Şenkul Ç, Kaya S (2017). Türkiye endemic bitkilerinin coğrafi dağılışı. Türk Coğrafya Dergisi 69: 109-120.

Tekşen M, Karaman Erkul S (2015). Gagea vanensis, a new species and G. chomutovae, a new record from Southeastern Anatolia, Turkey (Liliaceae). Phytotaxa 188(5): 251-260.

The International Plant Names Index (IPNI)http://www.ipni.org/index.html / [Accessed 16. May .2018].

The Plant List Version 1.1 (2018). http://www.theplantlist.org/ [Accessed 16 May 2018].

Torlak H, Vural M, Aytaç Z (2010) Endemic plants of Turkey. Ministry of Culture and Tourism Central Directorate of Revolving Funds, Ankara: PelinOfset.

Turkish Plants Data Service (TÜBIVES) Version 2.0 beta 2018: http://www.tubives.com/ [Accessed 16 May 2018].

Vitek E, Yüce E, Ergin C (2014). Gundelia dersim and Gundelia munzuriensis (Compositae), two new species from Turkey, Phytotaxa 161 (2): 130-138.

Yıldırım H, Bingöl Ö, Armağan M (2011). Crepis gemicii sp. nov. (Asteraceae) from east Anatolia, Turkey. Nordic Journal of Botany 29: 14-19. 
Demirkuş et al - Endemic plants ...

Yıldırırm H (2014). Puschkinia bilgineri(Asparagaceae alt familyaScilloideae): Doğu Anadolu Bölgesi’nden yeni bir Karsümbülü (Puschkinia Adams) türü. Bağbahçe Bilim Dergisi 1(1) : 1-10.

Yıldız B, Arabacı T, Dirmenci T, Çelenk S (2011). Cirsium sivasicum sp. nov. and C. peshmenianum sp. nov.(Asteraceae) and their allies from Turkey. Nordic Journal of Botany 29: 26-37.

Cite this article: Demirkuş N, Koyuncu M, Gül M (2018). Endemic plants of Lake Van Basin. Anatolian Journal of Botany 2(2): 70-83. 